\title{
Study on Access Selection of Heterogeneous Wireless Network in the View of Evolutionary Game
}

\author{
Longfei Guo ${ }^{1, *}$, Tingjie $\mathrm{Lv}^{2}$, Xue $\mathrm{Jin}^{2}$ and Xia Chen ${ }^{2}$ \\ ${ }^{1}$ Key Laboratory of Information Management and Economy, Beijing University of Post and Telecommunication, Beijing, \\ 100876, China \\ ${ }^{2}$ Beijng University of Post and Teleommunication No.10 Xitucheng Road, Haidian District, Beijing, Postcard 100876, \\ China
}

\begin{abstract}
Along with the continuous development of wireless communication technology and popularization of multimode terminal, the seamless fusion of various heterogeneous wireless networks has gradually become the trend of network development. The diversified forms of network access enable the multi-mode terminal to dynamically select the access network with the highest utility value to obtain network service according to specific standard. The study selects a typical heterogeneous network scenario, abstracts users' network selection process to be group game model, adopts the evolutionary methods, and then studies user's network selection process through replicator dynamics. On the basis of it, it puts forwards a kind of network selection algorithm in the view of replicator dynamics and verifies its algorithm performance through simulation results, and then analyzes the impact of different factors on evolutionary equilibrium in detail.
\end{abstract}

Keywords: Evolutionary equilibrium, evolutionary game, heterogeneous wireless network, network selection algorithm, replicator dynamics.

\section{INTRODUCTION}

Along with the rapid development of wireless communication technology, the heterogeneous network integrating a variety of wireless access technologies becomes the new pattern of wireless network, and the pattern that users has relied on a network for long is gradually being broken. In heterogeneous wireless network, various networks vary in the coverage, bandwidth, price, access speed, network capacity, etc. Thus, seamless vertical switching between different networks in accordance with user's demands, user service quality optimization and network load balancing have become crucial for the study on selection of heterogeneous wireless network.

In the environment of heterogeneous network, the load balancing of network can optimize network performance, avoid network congestion and deterioration, make the most of heterogeneous wireless network resource, increase system capacity and improve user perception. At the current stage, the load balancing is mainly realized in two ways, network drive or user drive. Network drive is controlled by network terminal, namely, service provider provides selection and decision-making. Network drive needs central processing controller which integrates the resources of different wireless networks, and uniformly allocates network resource to a particular area. The method needs tight integration of resources, so it signifies great signalling overheads at the same time. And user drive is realized in a distributed manner for network selection, without modification and coordination in different networks. This method is realized on the terminal side, with low complexity and less additional overheads, so it is easier to be realized relatively. But the terminal users shall get information for decision-making.

The study adopts the method of user drive. Based on evolutionary game, it builds models according to the dynamic selection of multiple user groups to study user's selection behaviour of dynamic network, and regard evolutionary equilibrium as the solutions to network selection. The study mainly focuses on the following:

1) Build basic heterogeneous network environment, and model user's network selection process according to user' different requirements for access network.

2) Simulate the network selection process into evolutionary game process, select the dynamic mechanism of replicator dynamics, study user's selection process, and regard the results of evolutionary equilibrium as user's network selection scheme.

3) Put forward the algorithm of evolutionary game of groups, reasonably set parameters, simulate the results, verify algorithm performance, and discuss in detail the impact of different factors on evolutionary equilibrium results.

\section{RELATED WORK}

Network access selection is one of key points of the next generation of heterogeneous converged network. Many liter- 
atures have carried out in-depth study on it. Kassar and Kervella [1] have summarized the hot access selection strategies in recent years, and classified and compared these strategies. Bari [2] considers that the current network selection algorithm does not take full factors into consideration, and puts forward a comprehensive decision-making method of candidate network ranking, including non-compensatory multi-attribute decision-making algorithm and compensatory multi-attribute decision-making algorithm. Stevens-Navarro [3] equalizes the access selection decision-making process as the markov decision process targeting at maximizing the total expected value.

Game theory, considered as a significant stool for study in many fields. It is also gradually used for study on heterogeneous wireless network in recent years.

Khan [4] introduces the concept of strategy space, solves network selection problems through the model of game theory, and provides the mathematical mechanism of network selection. Charilas D and Markaki O [5] have studied the wireless resource management mechanism in the heterogeneous environment, put forward the converged network selection mechanism and theoretical scheme of game theory, and modelled the access control into non-cooperative game, so as to get the maximum return through mutual game between networks, and then ensure to maximize service QoS. Zhu Kun [6] solves the selection problem through Bayesian game under the condition of incomplete information. During the study on dynamic selection, it introduces the best response dynamics Bayes and aggregates the best response dynamics, and verifies that, the balanced network selection for mobile users can be realized in case of incomplete information. Xingwei Liu [7] applies bidding game model to solve heterogeneous network switching problems; simulates the behaviour between user and network into bidding model of various tenderers, and also simulates the competitive behaviour between user and network into cooperative game. The mobile user selects the most suitable network to satisfy the demand of various applications QoS, and networks realize load balancing through mutual cooperation. Jianwei Huang [8] puts forward the pricing model based on auction game, used for distribution of network resources. The article also discusses the impact of upset price weighing the best network performance and income.

However, network selection is a dynamic problem as well as a group problem. In order to get the overall best performance, the users in groups require multiple games to continuously adjust strategies. Therefore, the evolutionary game with discussion on dynamic evolution is introduced to solve the problem of network access.

Literatures $[9,10]$ use evolutionary game for study on dynamic network access process. Niyato D and Hossain $E^{[9]}$ compare and analyze the network selection scheme and Nash equilibrium obtained from classical non-cooperation game model. Jiang Yong [10] proves the steady state of system evolution is Nash equilibrium, and all users obtain the optimal strategy solution with the same utility.

The study analyzes the network access process of bounded rationality and incomplete information through evolution- ary game theory. Users act as the game participant, and their selection of network act as strategy set. Then the users of different strategy sets form different groups. Based on user drive, it conducts evolutionary modelling for dynamic selection of multiple user groups, so as to make a study on user's dynamic network selection behaviour and evolutionary equilibrium, and also focus on the influence factor of net utility convergence process.

\section{EVOLUTIONARY GAME AND REPLICATOR DY- NAMICS}

\subsection{Evolutionary Game}

The traditional game theory usually assumes that participants are entirely rational under the condition of complete information. But in reality, the condition is difficult to realize. In heterogeneous network environment, users have target rationality, but they show limited cognitive competence and lack of previous decision-making behaviours of other users to predict the existing behaviours when faced with complicated, diversified and uncertain environment. Hence, compared with the entirely rational assumption in traditional game theory, it is more practical to consider users to be with limited rationality.

Evolutionary game, also called bounded rationality game, differs with the traditional game theory, for the complete rationality of participants and the condition of complete information are not required. Thus, the study adopts evolutionary game as analytical method for the study on network selection in heterogeneous network environment. The evolutionary game relaxes the requirements for participants of strict rationality, and analyzes how the participants with bounded rationality achieve a stable equilibrium state through learning process.

The equilibrium of bounded rationality is adjusted and improved in a continuous manner rather than the results of one-time selection. With a view to realizing the optimal performance with the minimum cost, users will redo the network selection decision-making in the wireless heterogeneous network until it achieves the equilibrium point. The equilibrium is the selection scheme for users to obtain the maximum return after giving the selection of other users. When the users achieve the equilibrium point, any user's changing of strategies unilaterally will not obtain more earnings.

\subsection{Replicator Dynamics}

The dynamic adjustment mode is the key point of evolutionary game. In case of various equilibriums in the system, which equilibrium can be achieved relies on the initial state and dynamic adjustment mode of evolution. Evolutionary game has two representative dynamic mechanisms.

Best-response Dynamics: Repeat game of small group members with rapid learning ability.

Replicator Dynamics: Immediately paired repeated game of large group members with slow learning. Replicator dynamics resembles the evolutionary process of biotic 


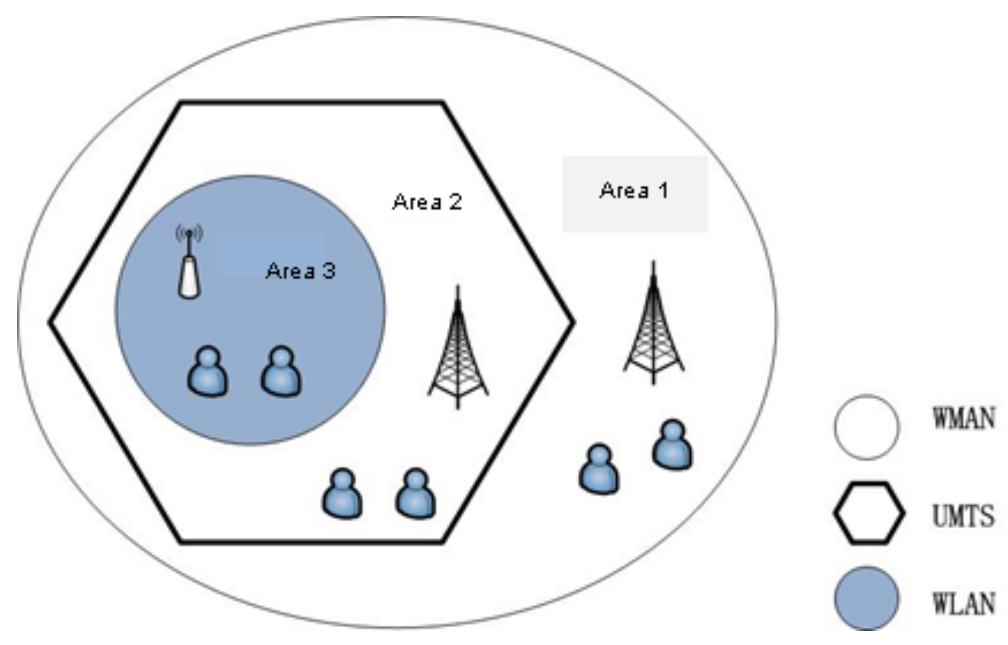

Fig. (1). Typical heterogeneous wireless networks .

population. During dynamic evolutionary game, a member of the group regenerates by selecting or mutating, which is called a replication process. The change of dominant strategy of game direction is a gradual process, rather than the simultaneous adjustment of all game directions. The speed of strategy adjustment can be expressed by evolutionary dynamic equation of biological evolution, the formula of replicator dynamics.

The study adopts the evolutionary game method of replicator dynamics equation to make a study on the selection mechanism of heterogeneous wireless network, for replicator dynamics can clearly present the process of network selection and is beneficial for analyzing the participants' network selection behaviours in the whole process.

\section{EVOLUTIONARY GAME MODEL OF HETERO- GENEOUS WIRELESS NETWORK}

\subsection{System Model}

The study is based on heterogeneous wireless network scenario, as shown in Fig. (1). It supposes that the user's terminal supports various network types, including WMAN, UMTS and WLAN.

The heterogeneous wireless network structure shown in Fig. (1) is very common in cities. In such heterogeneous wireless network environment, the area of wireless network coverage includes three areas: in Area 1, there is only WMAN (wireless metropolitan area network), so users here can have access to the network through WMAN; in Area 2, there is also UMTS (cellular network) covered, so users here can have access to network through WMAN and UMTS; similarly, users in Area 3 can have access to network through WMAN, UMTS and WLAN.

\subsection{Factors of Evolutionary Game}

Participants: The users with the right to select network are participants of the evolutionary game in the network coverage area of Fig. (1). Users in Area 2 and 3 where many heterogeneous networks cover can select access network; users in Area 1 are not able to participate in the game, because they can only select WMAN network access. From the perspective of evolutionary game, users in Area 2 and 3 constitute two groups respectively.

Strategy: Within the area covered by heterogeneous wireless network, the network selection of every participant is strategy in fact. For example, the strategy set of Area 3 is \{WMAN, UMTS, WLAN\}. Users in different areas can select a variable number of networks.

Income: The income of every participant depends upon the net utility and every participant tries to increase the net utility during evolutionary game till the evolutionary equilibrium is realized.

When all users in the same group obtain the same income in evolutionary game, they will not continue seeking for more income, thus their access network will not be changed any more. Then it is called evolutionary equilibrium.

In order to simplify calculation, the study assumes that only one class of service is for the same access network, namely, the service speed is the same for the users with same access network.

For the access network $\mathrm{k}$, its total system capacity is $C_{k}$, and the number of users who have access to network $\mathrm{k}$ at some point is $N_{k}$. Then the service quality obtained by single user is $Q_{k}=C_{k} / N_{k}$. The utility function is positively correlated with service quality of users who using network $\mathrm{k}$ and the utility is $U\left(Q_{k}\right)$. Assume the price function $P\left(N_{k}\right)$ of network $\mathrm{k}$ progressively increases with the increasing number of users, because the increase in number of single network users will aggravate the network congestion and rise in price of service is helpful to reduce congestion. In conclusion, the net utility of users who have access to network $\mathrm{k}$ is:

$E_{k}=U\left(C_{k} / N_{k}\right)-P\left(N_{k}\right)$ 
Introduce formula (1) into the heterogeneous wireless network shown in Fig. (1), and then the net utility of users who have access to WMAN network can be obtained

$$
E_{w m}^{1}=U\left(C_{w m}^{1} / N_{w m}\right)-P\left(N_{w m}\right)
$$

In which, $N_{w m}=N_{w m}^{1}+N_{w m}^{2}+N_{w m}^{3}$, namely, the number of users selecting WMAN is the total number of users selecting the network in three areas.

\subsection{Replicator Dynamics}

In evolutionary game, single user observes the income of other users in same group in every stage and adopts some strategies for more income in the next stage. The speed for users to change strategy is expressed with parameter $\sigma$ and then the formula of replicator dynamics is:

$\dot{x}_{k}^{a}=\sigma x_{k}^{a}\left(E_{k}^{a}-\bar{E}^{a}\right)$

In which, $\bar{E}^{a}=\sum_{k} x_{k}^{a} E_{k}^{a}$ refers to the average value of net utility of all users in Area a. It can be known from formula (3) that if the net utility of network $\mathrm{k}$ is greater than the average net utility, the number of users who have access to network $\mathrm{k}$ will increase, the net utility of single user will reduce and then network selection develops towards evolutionary equilibrium. According to the structure of formula (3), it can be inevitably drawn that: $\sum_{k} \dot{x}_{i}^{a}=0$, and replicator dynamics satisfies the equation in whole process.

Apply formula (3) in the heterogeneous wireless network shown in Fig. (1), it can be obtained that:

$$
\begin{gathered}
\dot{x}_{w m}^{2}=\sigma x_{w m}^{2}\left(E_{w m}^{2}-x_{w m}^{2} E_{w m}^{2}-\left(1-x_{w m}^{2}\right) E_{u m}^{2}\right) \\
\dot{x}_{w m}^{3}=\sigma x_{w m}^{3}\left(E_{w m}^{3}-x_{w m}^{3} E_{w m}^{3}-x_{u m}^{3} E_{u m}^{3}\right. \\
\left.-\left(1-x_{w m}^{3}-x_{u m}^{3}\right) E_{w l}^{3}\right) \\
\dot{x}_{u m}^{3}=\sigma x_{u m}^{3}\left(E_{u m}^{3}-x_{w m}^{3} E_{w m}^{3}-x_{u m}^{3} E_{u m}^{3}\right. \\
\left.-\left(1-x_{w m}^{3}-x_{u m}^{3}\right) E_{w l}^{3}\right)
\end{gathered}
$$

\subsection{Evolutionary Equilibrium}

There will be a time point in the process of replicator dynamic equation, when users in the same group have the same net utility. For any $k$ or $a, \dot{x}_{k}^{a}=0$ can be obtained on the basis of formula (3), namely, single user will not try to increase its net utility by changing the network. Therefore, the equilibrium point in the evolutionary game can be obtained through the solution of $\dot{x}_{k}^{a}=0$.

\section{NETWORK SELECTION ALGORITHM AND SIM- ULATION}

\subsection{Network Selection Algorithm}

The study puts forward a kind of network selection algorithm based on group evolutionary game. And a controller is required to control the net utility of all users in the group with certain characteristics, and then calculate the average utility value of the group; in the next stage, users in group will change network selection strategy according to the average utility value. The specific selection algorithm is to be mentioned as follows:

1) All users randomly select usable access network (WMAN, UMTS, WLAN) in the initial stage

\section{(2) Circulation}

Single user calculates net utility $E_{k}^{a}$ according to the bandwidth distributed and sends the net utility information to central control unit;

The central control unit calculates the average net utility $\bar{E}_{k}^{a}$ within the group and then sends it to the users in group;

If $\bar{E}_{k}^{a}>E_{k}^{a}$, select another network j, satisfying $E_{j}^{a}>E_{k}^{a}$ and $j \neq k$;

\section{3) End of circulation}

\subsection{Simulation Result and Analysis}

Assume that the number of users in the three areas is $N^{1}=10, N^{2}=20$ and $N^{3}=30$ respectively in the heterogeneous network shown in Fig. (1). According to the standard of communication agreement, the actual velocity of WLAN of 802.11 standard is about $7 \mathrm{Mb} / \mathrm{s}$, the community velocity of WCDMA of R99 version in UMTS reaches $2 \mathrm{Mb} / \mathrm{s}$, and the velocity of WMAN of 802.16 standard is $10 \mathrm{Mb} / \mathrm{s}$.

In terms of the utility function and price function of formula (1), for the convenience of calculation, the utility function shall be defined as $U(x)=x$, and $x$ as the distributed bandwidth; price function as $P\left(N_{k}\right)=N_{k} / 100$ and $N_{k}$ as the total number of users selecting network $\mathrm{k}$ in one area.

(1) Selection process of replicator dynamics

Assume that no users select UMTS in Area 3, then the network selection strategies that may be adopted in the three areas are WMAN, WMAN and UMTS respectively; for WMAN and WLAN, the speed for users to change strategy is $\sigma=2$. Based on the above parameter setting, and by adopting the network selection algorithm put forward in Section 5.1 and tracing the dynamic network selection behaviours dynamically, then the result shown in the following Figure can be obtained:

Fig. (2) shows that starting from specific network distribution and conducting network dynamic selection through replicator dynamics, the evolutionary equilibrium is realized finally after 10 replication processes. It can be known from the analysis of Fig. (2) that no matter how the users in Area 2 and 3 are distributed at initial state, their network selection distribution tends to be stable after limited replicator dynam-

ics, and then the replicator dynamics of formula (3) is $\dot{x}_{k}^{a}=0$ , namely, no users in Area 2 and 3 change the access network 


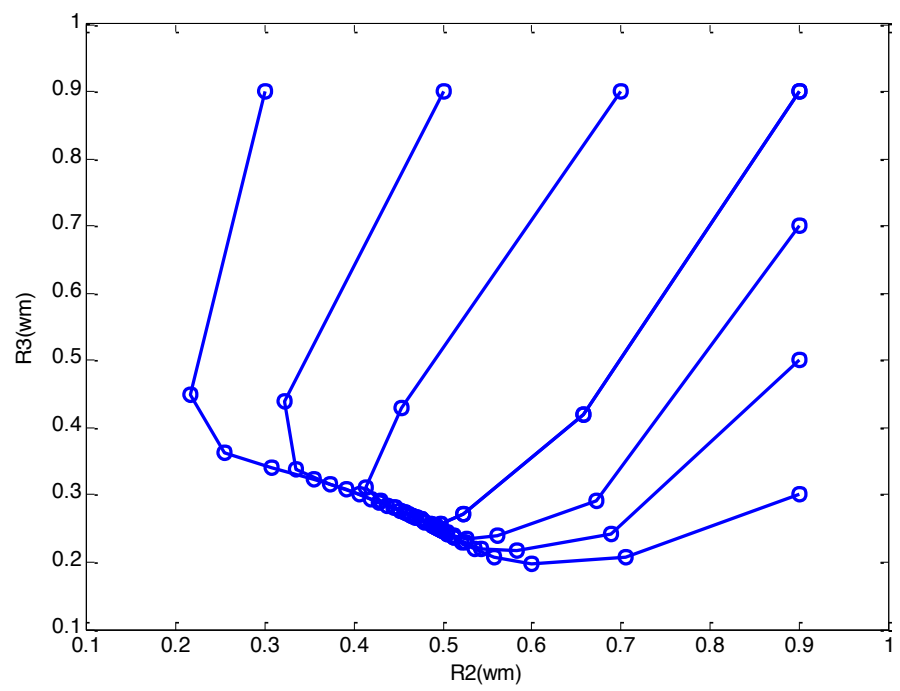

Fig. (2). Process of replicator dynamics.

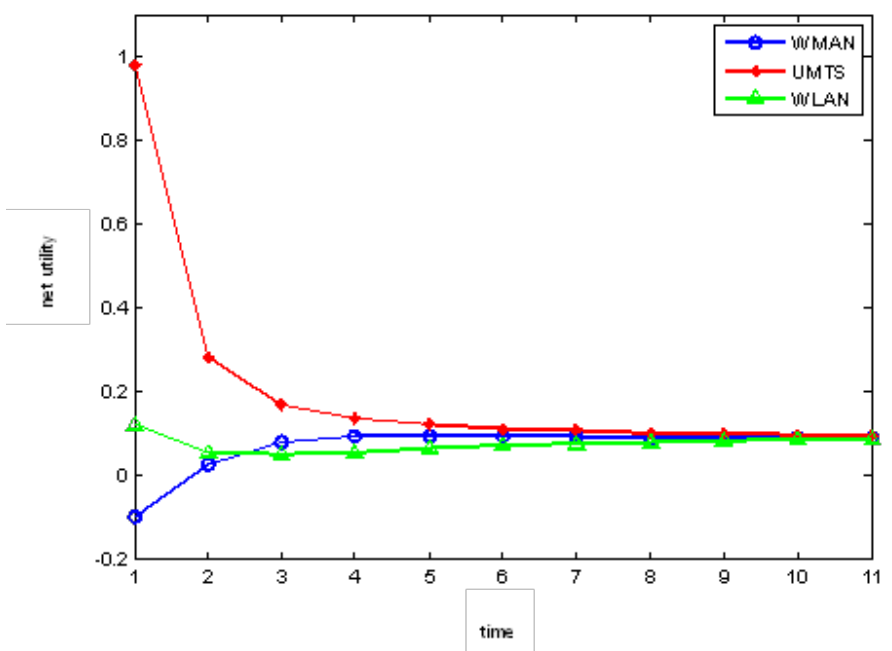

Fig. (3). $\sigma=2$ Convergence process of network net utility.

for obtaining more net utility and then evolutionary equilibrium is realized. With the parameter setup, when evolutionary equilibrium is reached, the result of equilibrium point is $[0.48,0.26]$, proportions of WMAN and UMTS users in Area 2 are $48 \%$ and $52 \%$ respectively and proportions of WMAN and WLAN users in Area 3 are $26 \%$ and $74 \%$ respectively. At the moment the net utility of users in Area 1, 2 and 3 is equal to their average net utility, namely, 0.09 .

Take the initial user distribution $[0.9,0.3]$ as example, namely, the proportion of users selecting WMAN and UMTS in Area 2 is 9:1, and the proportion of users selecting WMAN and WLAN in Area 3 is 3:7 in initial state. Figure 3 displays the net utility change of three different access networks in 10 processes of replicator dynamics.

In Fig. (3), the net utility of three networks tends to be converged till the tenth replication and then heterogeneous network reaches evolutionary equilibrium.
(2) Impact of replication speed on equilibrium

To discuss the impact of replication speed on the convergence of net utility, two Figures for replicator dynamics speed ( $\sigma=1$ and $\sigma=5$ ) are drawn, namely, Fig. (4 and 5).

It can be obtained after the analysis of Fig. (4) that when $\sigma=1$, users in the area change network selection more slowly till the $19^{\text {th }}$ replication process, and then the net utility value of three networks starts to be converged and heterogeneous network reaches evolutionary equilibrium. And at the moment, the net utility value is equal to the result of Fig. (3), but it takes twice the time.

It can be analyzed from Fig. (5) that when $\sigma=5$, users in the area change network more quickly and the average net utility value of network changes greatly in every replication process. However, after a long time, the net utility value of three networks can still be not converged and remains 


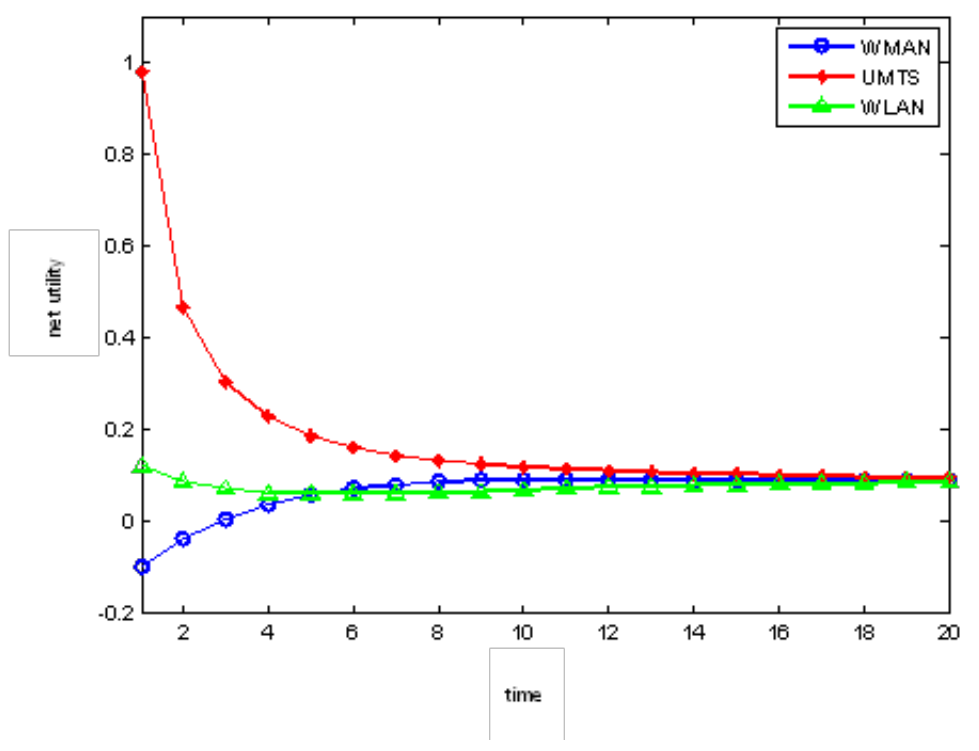

Fig. (4). $\sigma=1$ Convergence process of network net utility.

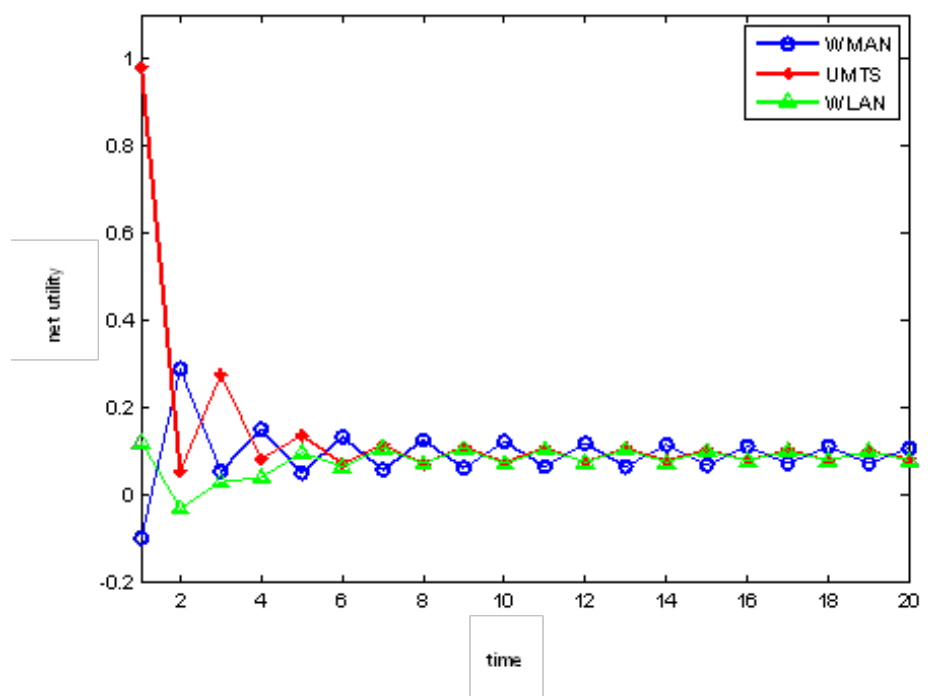

Fig. (5). $\sigma=5$ Convergence process of network net utility.

around the original equilibrium point. Therefore, heterogeneous network cannot realize evolutionary equilibrium.

It can be known from the above analysis that replication speed $\sigma$ plays an important role in study on the selection process of heterogeneous network by adopting the method of dynamic replication: in case of low speed, it needs to take a long time to realize evolutionary equilibrium as few users make adjustment for each replication, and every replication process requires the information interaction with network controller, thus causing unnecessary waste of resources; in case of high speed, there are many users changing their network selection strategy for each replication, but after a long time, the network still cannot realize evolutionary equilibrium and remains around the equilibrium point. On the one hand, the frequent network switching of lots of users occupies a great number of signalling resources; one the other hand, for the evolutionary equilibrium cannot be realized, bandwidth resource is wasted. Therefore, when selecting the speed of dynamic replication, the selection of speed range shall be based on actual situation, avoiding wasting resources.

\section{(3) Impact of number of users on equilibrium}

With other conditions not changed, assume the number of users in Area $3 N^{3}$ changes, then it can be seen from the simulation result that the number of users exerts influence on bandwidth and price and the corresponding evolutionary equilibrium point also changes. The simulation result is shown as in Fig. (6-8). 


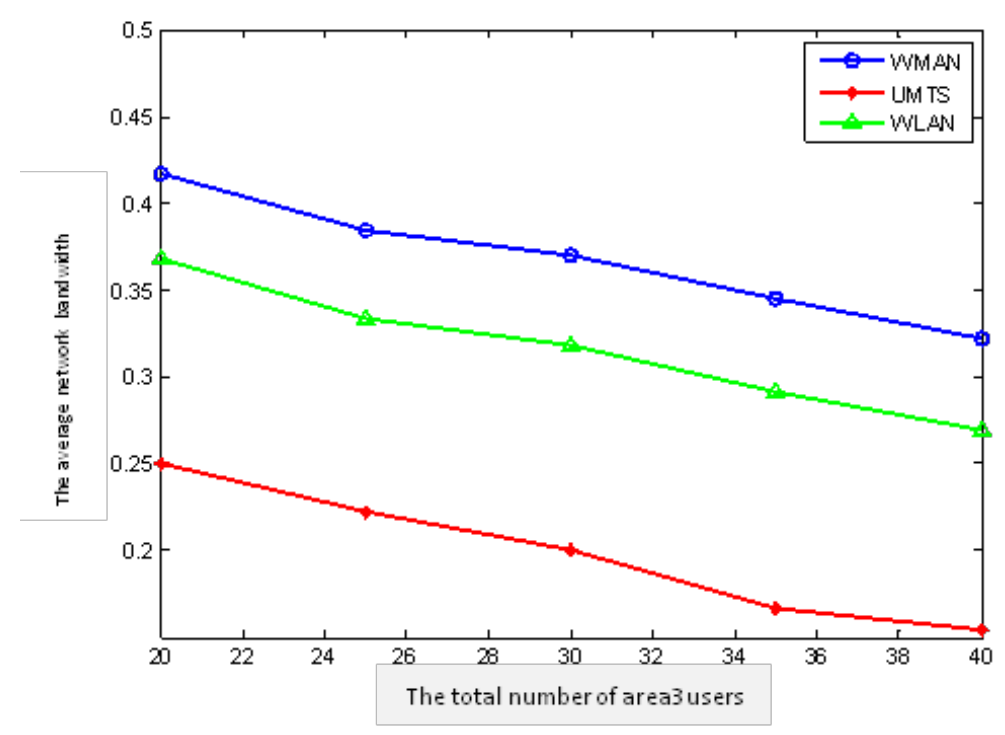

Fig. (6). Impact of number of users in area 3 on bandwidth.

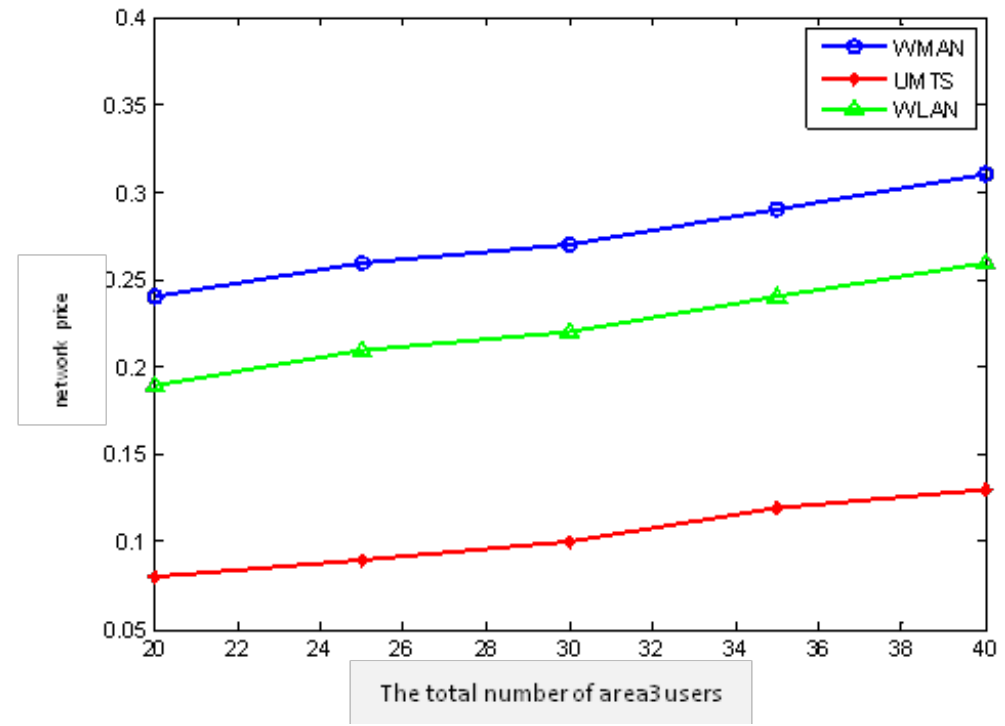

Fig. (7). Impact of number of users in area 3 on price.

It can be known from the above three Figures that the change of number of users in single area exerts influence on the equilibrium of the whole area, which is reflected in bandwidth, network price and net utility of the three areas. When there are increasing users in Area 3, the load of WMAN and WLAN within Area 3 increases, thus the network net utility in the area declines sharply, bandwidth reduces and price rises. The net utility decline of WMAN will affect Area 2 and 1, which results in the increasing number of users selecting UMTS and acts on bandwidth and price at the same time. As shown in the Figure, three networks are in equilibrium state finally, but their net utility is at a lower level.

Thus it can be seen that the increasing users in any area will lead to a drop in service quality of whole heterogeneous network and meanwhile, aggravate the network load and accordingly cause a rise in price of network access. However, there is still a positive correlation relationship between price and bandwidth, namely, WMAN with the best service quality charges the highest fees.

\section{CONCLUSION AND OUTLOOK}

The study selects a typical heterogeneous network scenario to abstract the network selection process as a group game model, and adopts the methods of evolutionary game and replicator dynamics to study the network selection process of users. The study designs network selection algorithm based on replicator dynamics, simulates and analyzes the game process of users and further discusses the key factors affecting equilibrium. 


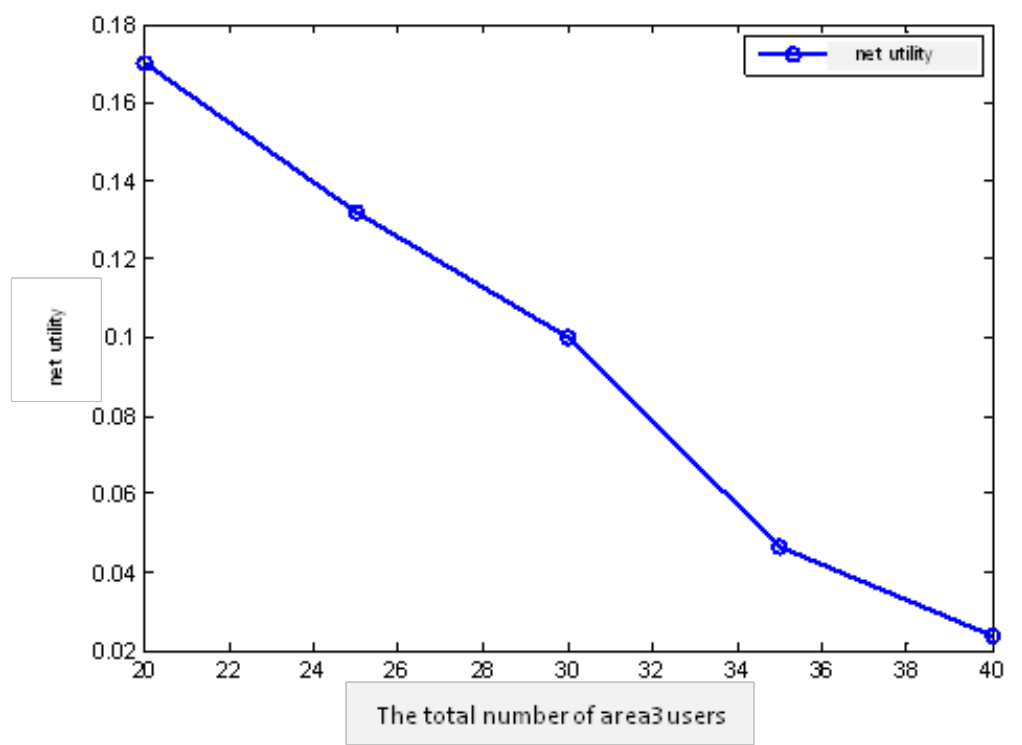

Fig. (8). Impact of number of users in area 3 on equilibrium net utility.

To make study and analysis process simple, the study simplifies the scenario of heterogeneous network. In future, the study will conduct further exploration on the evolutionary equilibrium under complex network structure.

\section{CONFLICT OF INTEREST}

The author confirms that this article content has no conflict of interest.

\section{ACKNOWLEDGEMENTS}

This Project was supported by National Basic Research Program of China (973 Program) under Grant No. 2012CB315805; National Natural Science Foundation of China under Grant No. 71172135 and No.71231002; "the Fundamental Research Funds for the Central Universities" (2013RC0603).

\section{REFERENCES}

[1] M. Kassar, B. Kervella and G. Pujolle, "An overview of vertical handover decision strategies in heterogeneous wireless networks," Computer Communications, vol.31, pp. 2607-2620, Jun. 2008.

[2] F. Bari and V.C.M. Leung, "Automated network selection in a heterogeneous wireless network environment," Network, vol. 21, pp. 34-40, 2007.
[3] E. Stevens-Navarro, Y. Lin and V.W.S. Wong, "An MDP-based vertical handoff decision algorithm for heterogeneous wireless networks," Vehicular Technology, vol. 57, pp. 1243-1254, Mar. 2008.

[4] M.S.Z. Khan, S. Alam and M.R.H. Khan, "A network selection mechanism for fourth generation communication networks," Journal of Advances in Information Technology, vol. 1, pp. 189-196, Nov. 2010.

[5] D. Charilas, O. Markaki and E. Tragos, "A theoretical scheme for applying game theory and network selection mechanisms in access admission control," Wireless Pervasive Computing, pp. 303-307, 2008.

[6] Z. Kun, N. Dusit and W. Ping, "Network Selection in Heterogeneous Wireless Networks Evolution with Incomplete Information," in Wireless Communications and Networking Conference, pp. 1-6, 2010 .

[7] X. Liu, X. Fang, X. Chen, "A bidding model and cooperative game-based vertical handoff decision algorithm," Journal of Network and Computer Applications, vol. 34, pp. 1263-1271, Jul. 2011.

[8] J. Huang, Z. Han, M. Chiang, "Auction-based resource allocation for cooperative communications," Selected Areas in Communications, vol. 26, pp. 1226-1237, Sep. 2008

[9] D. Niyato and E. Hossain, "Dynamics of network selection in heterogeneous wireless networks an evolutionary game approach," IEEE Transactions on Vehicular Technology, vol. 58, pp. 20082017, May 2009.

[10] J. Yong, H. Bo and C. Shanzhi, "Network correlation optimization of heterogeneous wireless network users: one method based on group game", Chinese Journal of Computer, vol. 35, pp. 12491261, Jun. 2012.

(c) Guo et al.; Licensee Bentham Open.

This is an open access article licensed under the terms of the Creative Commons Attribution Non-Commercial License (http://creativecommons.org/licenses/by-nc/3.0/) which permits unrestricted, non-commercial use, distribution and reproduction in any medium, provided the work is properly cited. 